\section{How does variation count?}

\section{Alice S. Huang and John M. Coffin}

WHAT do virologists, evolutionary biologists, population geneticists, mathematical modellers, epidemiologists and physicians have in common? At a recent EMBO workshop* an area of overlap emerged that could grow into a powerful scientific interface. New approaches were reported for analysing viral disease processes and evolutionary relationships, in which computer simulations were combined with the wealth of molecular data being generated by the sequencing of viral genomes. Complex but manipulatable experimental viral systems were used to test theories of population dynamics. And, almost as an aside, it was firmly established that all RNA viruses, not only human immunodeficiency virus, mutate - but do not necessarily evolve - at a high rate, and that recombination is relatively common among RNA viruses.

Ever since von Magnus described changes in influenza virus populations almost 40 years ago, investigators have sought the cause of these changes. They have wondered whether such variations, so easily detected in cell culture systems,

*EMBO Workshop on Variation and Molecular Evolution of Viruses, Grignon, France. 7-11 July 1992. could account for altered virulence in the infected host. Sparked by what seems to be a remarkable genetic plasticity among human immunodeficiency virus and the annual emergence of threatening new influenza virus strains, a closer inspection was made of what is currently known about mutation rates for viruses. Rates of mutation for RNA genomes are now estimated to be 1,000-fold higher than for DNA genomes (J. Drake, National Institute of Environmental Health Sciences). Furthermore, the rates are highly variable from site to site in the same genome (T. Kunkel, National Institute of Environmental Health Sciences; B. Preston, Rutgers; M. Ricchetti, Pasteur Institute). This microheterogeneity leads to a population of viral genomes known as quasispecies, which are closely related but different from one another. The proportions of these quasispecies change, depending on both intrinsic error rates in polymerases and selective forces that may vary according to the host environment. From the composition of different quasispecies, viral replication rates, fitness selection and mutation rates can all be estimated in that virus population
(M. Nowak, University of Oxford)

Using improved molecular technology, particularly the ready amplification of nucleic acid sequences by the polymerase chain reaction, mutational heterogeneity is easily detected among different specimens taken at the same time and place from different patients, and in sequential specimens taken from the same patient. Although genetic variation may itself be important for pathogenesis, the immune system provides an important selective force ( $R$. Zinkernagel, University of Zurich; R. Phillips, John Radcliffe Hospital, Oxford; S. Carpenter, Iowa State University; A. Leigh-Brown, University of Edinburgh). Disappointingly, there were no clear-cut correlations between the degree of heterogeneity and the course of disease. It may well be that genetic change among viruses is intrinsic and the number of mutations simply related to the number of replication cycles (S. Wain-Hobson, Pasteur Institute), resulting in a genetically diverse population capable of responding to very small selective forces.

Besides the heterogeneity resulting from single base mutations, a range of strange and wonderful variations among viruses was presented. These included satellite viruses of plant and animal viruses, where there is no commonality of sequence between the parasite and its

\title{
Lifting the lid on enzymatic breakdown of lipids
}

LIPASES are enzymes that hydrolyse triacylglycerol lipids into free fatty acids and glycerol - a crucial step in breaking down ingested fats in the gut. The pancreatic lipase enzyme's activity is greatly increased at the lipid-water interface, when a short amphipathic helix, or lid (A), in the amino-terminal domain of the lipase (B), pops open and exposes the active site (C) of the enzyme to its lipid substrate (a phenomenon known as interfacial activation). The interaction of the lipase enzyme with the lipid interface is very sensitive to the chemical nature of the lipid surface; in the presence of amphiphiles such as bile salts and phospholipids, the binding of the lipase to the lipid surface requires a small protein cofactor, colipase. The complex of these two molecules reveals that colipase binds to the carboxyterminal domain of the pancreatic lipase molecule (see the paper by $H$. van Tilbeurgh et al. on page 159 of this issue). The colipase's three hydrophobic fingers (F), here ball-and-stick groups, are sample hydrophobic amino-acid side chains that point away from the lipase molecule but are on the same face of the complex as the lid that covers the active site of the lipase molecule. The binding of lipase, but not colipase, to the lipid-water interface is inhibited by amphiphiles (like bile salts). In the

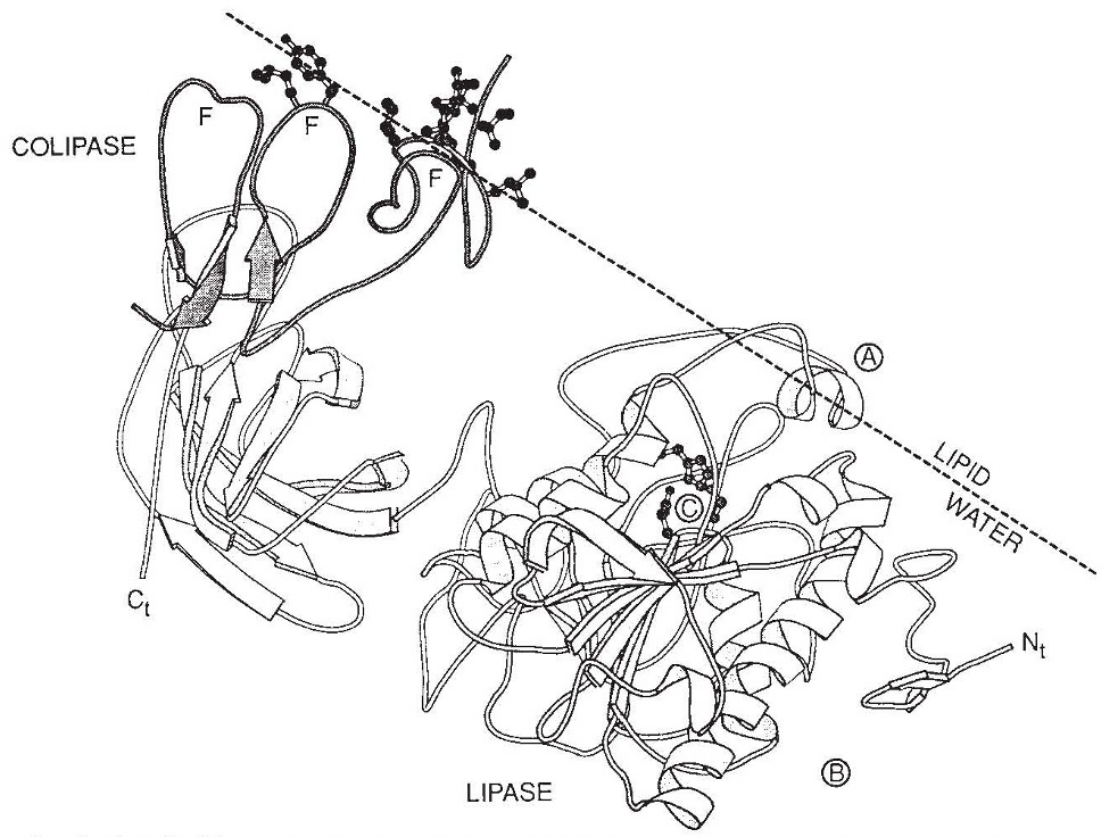

presence of such inhibitors, then, the colipase cofactor may bring the larger lipase enzyme into close contact with the interface and allow opening of the lid to expose the active site, so facilitating the enzymatic breakdown of lipids. (Figure adapted from P. J. Kraulis J. appl. Crystallogr. 24, 946-950; 1991.) 